\title{
Wall Following and Human Detection for Mobile Robot Surveillance in Indoor Environment
}

\author{
Chi-Wen Lo Kun-Lin $\mathrm{Wu}$ and Jing-Sin Liu \\ Institute of Information Science, Academia Sinica, Taiwan \\ liu@iis.sinica.edu.tw
}

\begin{abstract}
This paper describes the design of an indoor surveillance system capable of wall following and human detection based on intelligent mobile robot navigation. The wall following is performed based on a differential velocity control using type-2 fuzzy logic for a wheeled mobile robot equipped with IR sensors and sonar sensors, and human detection is performed by a human detection sensor. We have tested the application of our design in mobile robot for an indoor surveillance task of a polygon terrain using right wall following. The surveillance system of mobile robot is effective to work well in our testing.
\end{abstract}

Keywords: Wall following, fuzzy logic controller, human detection, surveillance

\section{INTRODUCTION}

Intelligent surveillance system using one or a team of autonomous mobile robots is increasingly popular and useful in civil and military applications such as home services, health care, border patrol to support the living safety of people [8][9]. Applying fuzzy logic control to design mobile robot controller has become a popular and useful method in mobile robot reactive navigation and control [2],[3],[10]-[11],[15]. Fuzzy logic theory has been widely applied to the design of controller because of the following reasons: 1) Control rules are more flexible and transparent; 2) The controller can emulate the human decision making; 3) It does not need a detailed or precise physical or mathematical model of the plant derived from first principles, and thus can simplify the control design process of unknown complex system. It replaces the mathematical values in describing control system by using the lingual ambiguous expression variables for designing controllers that are able to deliver a satisfactory performance in face of uncertainty and imprecision. Many papers demonstrated that the performance of type-2 fuzzy system is much better than type-1 fuzzy system [15], [2]. The antecedent in each fuzzy rule uses a type-2 fuzzy set to enhance the robustness [16].

Wall following is a method used by bugs and reactive mobile robot navigation to escape from local minima [1]-[7], [12], [18]. This research presents an autonomous wall following on indoor environment with the detection of human motion to support the mobile robot navigation task of surveillance using only local information of the environment gathered by sensors embedded on mobile robot. We considered the energy consumption problem of a battery- powered autonomous mobile robot. Our main objective of control is to design a velocity controller for reduce energy consumption [17]. In this paper, design of fuzzy logic controller for wall following of wheeled mobile robot is presented. Our system has a single fuzzy controller equipped with various sensors, IR sensors, human detection sensor and sonar sensors that each of them has many inputs from sensors. The distance of robot from wall or obstacles is detected by IR and sonar sensors. In our work, the robot is equipped a human motion sensor so that it could be used in indoor environment to detect human behavior, and the technique is novel and foresight [13] [14].

This paper is presented as follows. Section II presents the control system of the mobile robot. Section III introduces the sensors used for wall following and human detection. Section IV presents a type-2 fuzzy controller structure including design. Experimental results of an indoor navigation testing are reported in Section V. Conclusion is made in Section VI.

\section{THE MOBILE ROBOT}

\section{A. Wheeled Mobile Robot}

We develop, design and test our control algorithm for surveillance on a commercially available mobile robot platform Dr. robot X80 [14]. The X80 robot is an integrated electronic and software robotic system, and available to the designer through a set of ActiveX control components (SDK) developed for $\mathrm{C}$, Matlab and LabView in this research. The robot offers full Wifi (802.11b) and uses a wide range of sensors including IR sensor, sonar sensor, human detection sensor for surveillance applications. Fig.1 (a) denotes the physical and sensor configurations of the mobile robot, and Fig. 1 (b) is the photo of the mobile robot.

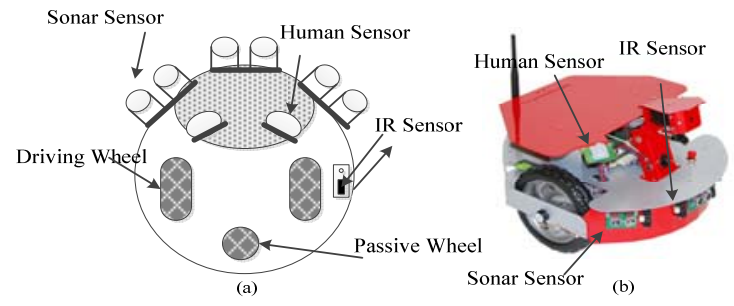

Fig. 1. The mobile robot: (a) the sensor configurations, (b) the photo.

B. Wireless Control System 
Fig. 3 shows the structure of the robot control system through wireless communication, which the navigation algorithm runs directly on the remote PC. We communicate with the robot and the computer by a serial wireless module, and the robot is equipped with a wide range of sensors to receive the information from real environment. The robot sensing and motion controller is designed, the around modules of the robot connectors to be operating by DSP processor as the interface device between between the remote PC, robot and sensors.

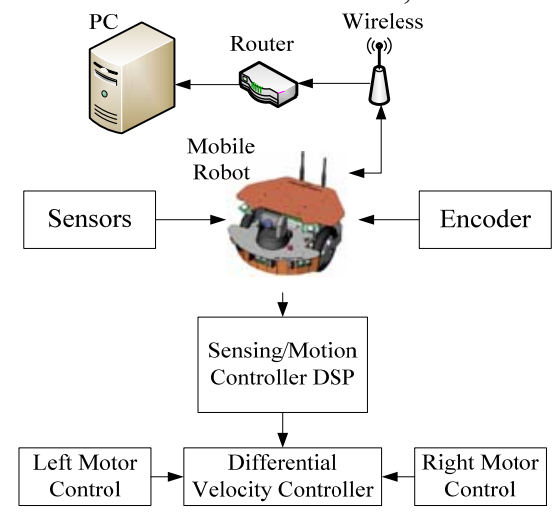

Fig. 3. Wireless control system. LabView program to control the mobile robot is on the remote computer.

\section{Drive System}

The motion of mobile robot is controlled by two DC motors of two wheels, where the differential velocity is used to drive moving direction. The robot motion coordinates chart is shown in Fig. 2. We consider the kinematic equality

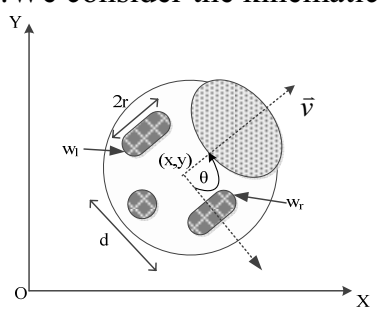

Fig. 3. The global and robot coordinate systems for robot motion

constraints are linear transform, which can be expressed as follows :

$\left[\begin{array}{l}v \\ w\end{array}\right]=\left[\begin{array}{ll}\frac{r}{2} & \frac{r}{2} \\ -\frac{r}{d} & \frac{r}{d}\end{array}\right]\left[\begin{array}{l}w_{l} \\ w_{r}\end{array}\right]$

where $w_{l}$ and $w_{r}$ denote initial left wheel velocity and initial right wheel velocity, $r$ is wheel radius, $d$ is distance between two wheels, $v$ and $w$ denote velocity and angular velocity. The detailed parameters of the mobile robot are listed in Table I. The real output is the speed commands for the robot wheels corresponding to a turning angle $\theta$

$V_{\text {Left }}=(d / 2) * \frac{\theta}{180} * \pi-w_{l}$

Table I

\begin{tabular}{|c|c|}
\hline Robot Parameters & Value \\
\hline Wheel Radius & $12.5(\mathrm{~cm})$ \\
\hline $\begin{array}{c}\text { Distance Between Two } \\
\text { Wheels }\end{array}$ & $25(\mathrm{~cm})$ \\
\hline Dimensions & $38(\mathrm{~cm}) \times 25.5(\mathrm{~cm})$ \\
\hline Weight & $3.5 \mathrm{~kg}$ \\
\hline Max Speed & $1 \mathrm{~m} / \mathrm{sec}$ \\
\hline Torque & $300($ oz.-inch $)$ \\
\hline
\end{tabular}

$V_{\text {Right }}=(d / 2) * \frac{\theta}{180} * \pi+w_{r}$

The initial velocity of two wheels is verified by the measurement, and the angle of rotation is send from the fuzzy controller. The block diagram of the generating velocities is shown in Figure 4.

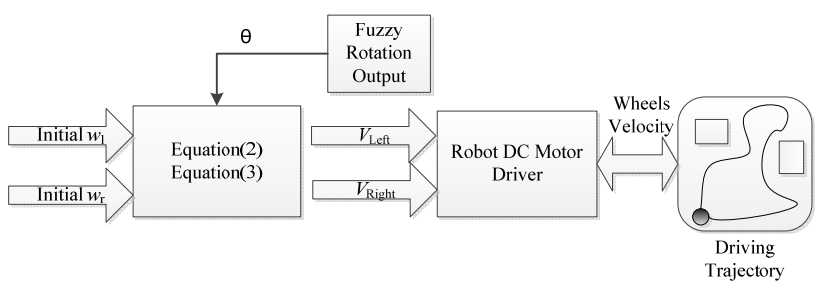

Fig. 4. The block diagram of generating velocities

\section{SENSORS}

A multisensory system composed by three types of sensors is embedded on the mobile robot for distance sensing and human detection, and does not impose computational burden for data processing.

\section{A. DUR5200 Ultrasonic Sensor}

The ultrasonic sensor can measure a distance range from 4 to $255 \mathrm{~cm}$, and the sound wave propagation speed $v$ can be calculated by following formula (4):

$v=331.5+0.6 * T[\mathrm{~m} / \mathrm{sec}]$

where $T$ is the air temperature $\left({ }^{\circ} C\right) . t_{d}$ value is calculated by count value (0 255) and count cycles of $80 \mathrm{us}$ times as follows (5):

$t_{d}=\operatorname{count}(0 \sim 255) *$ count_rate $(80 u s)$

The distance to object (in meter) can be obtained as follows (6):

Distance to object $=\left(t_{d} * v\right) / 2$

\section{B. GP2Y0A21YK Sharp IR Sensor}

The IR sensor detects a distance range from 10 to $80 \mathrm{~cm}$. We use distance output type (analog voltage) following adjustment and output switching distance must be measured by sensor, and verse distance to reflective object. 


\section{Human Motion Sensor}

In a real indoor environment, the objects including pet animals and human may appear abruptly. These living objects generate heat also generate infrared radiation. The human motion sensor has two sensing elements, one left and one right on the mobile robot (Fig. 1) that can detect the presence or absence of a human near the mobile robot. Fig. 4 illustrates the human detection using the human sensor detection.

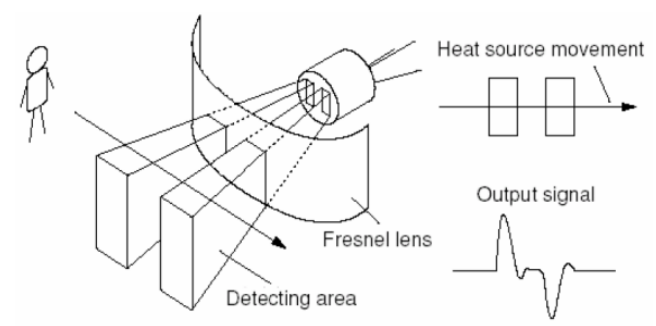

Fig. 4. Human presence or absence detection

\section{INTELLIGENT CONTROLLER STRUCTURE}

\section{A. Type-2 Fuzzy Logic System}

A type-2 fuzzy set $\widetilde{A}$, is characterized by the membership function as follows (7).

$$
\widetilde{A}=\left\{\left((x, u), \mu_{\widetilde{A}}(x, u)\right) \mid \forall x \in X, \forall u \in J_{x} \subseteq[0,1]\right\}
$$

In fact $J_{x} \subseteq[0,1]$ represents primary membership of $x$, and $\mu_{\widetilde{A}}(x, u)$ is a type-1 fuzzy set. Uncertainty is represented by a region, which is called the footprint of uncertainty (FOU). When $\mu_{\widetilde{A}}(x, u)=1, \forall u \in J_{x} \subseteq[0,1]$, we have an interval type-2 membership function, as shown in Fig. 5.

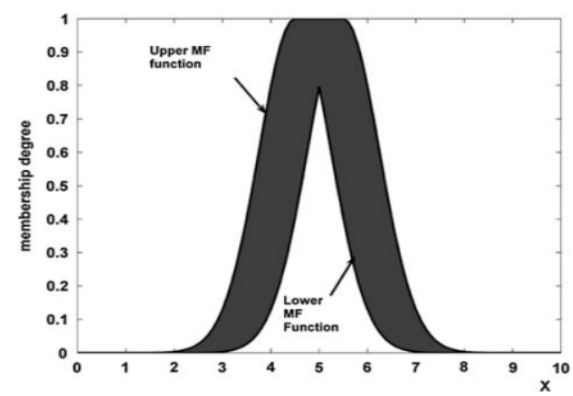

Fig. 5. Interval type-2 membership function

The type-2 fuzzy logic system is shown in Fig. 6. It includes a fuzzifier, a rule base, and a fuzzy inference.

1)Fuzzifier: The fuzzifier maps a numeric vector $x=\left(x_{1}, \ldots, x_{p}\right)^{T} \in X$ into a type-2 fuzzy set $\widetilde{A}_{x}$ in $X$, an interval type-2 fuzzy set in this case.
2)Rules: A type-2 fuzzy logic system with $p$ inputs (linguistic variables), $x_{1} \in X_{1}, \ldots x_{p} \in X_{p}$ and one output $y \in Y$, and

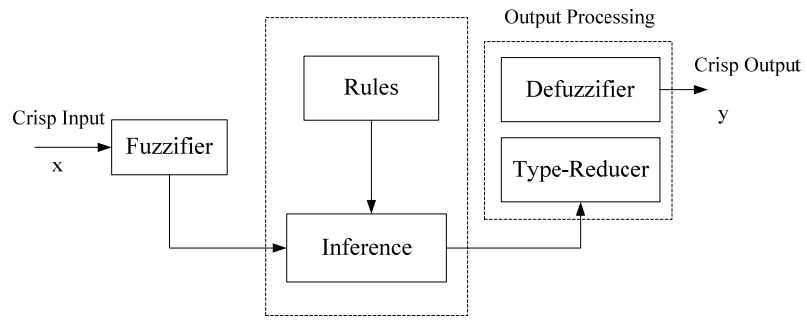

Fig. 6. Type-2 fuzzy logic system

we assume $M$ rules, the $l$ th rule in the system can be written as follows (8).

$$
\begin{aligned}
& R^{l}: I F x_{1} \text { is } \widetilde{F}_{1}^{l} \text { and } \cdots \text { and } x_{p} \text { is } \widetilde{F}_{p}^{l}, \\
& \text { THEN } y \text { is } \widetilde{G}^{l} l=1, \ldots, M
\end{aligned}
$$

where the $F$ and $G$ are appropriate fuzzy sets for each rule.

3)Inference: If $\widetilde{F}_{1}^{l} \times \cdots \times \widetilde{F}_{p}^{l}=\widetilde{A}^{l}$, can be written as follows (9).

$R^{l}: \widetilde{F}_{1}^{l} \times \cdots \times \widetilde{F}_{p}^{l} \rightarrow \widetilde{G}^{l}=\widetilde{A}^{l} \rightarrow \widetilde{G}^{l} l=1, \ldots, M$

$R^{l}$ is described by the membership function $\mu_{R^{l}}(x, y)=\mu_{R^{l}}\left(x_{1}, \ldots x_{p}, y\right)$, where

$\mu_{R^{l}}(x, y)=\mu_{\widetilde{A}^{l} \rightarrow \widetilde{G}^{l}}(x, y)$ can be written as (10).

$\mu_{R^{\prime}}(x, y)=\mu_{\widetilde{A}^{\prime} \rightarrow \widetilde{G}^{l}}(x, y)=\mu_{\widetilde{F}^{l}}\left(x_{1}\right) \prod \cdots \prod \mu_{\widetilde{F}_{p}}\left(x_{p}\right) \prod \mu_{\widetilde{G}^{l}}(y)$

$=\left[\prod_{i=1}^{p} \mu_{\widetilde{F}_{i}^{l}}\left(x_{i}\right)\right] \prod \mu_{\widetilde{G}^{l}}(y)$

In this system, we use interval type-2 fuzzy sets and intersection under product t-norm, which are contained in the firing set $\prod_{i=1}^{p} \mu_{\widetilde{F}_{i_{i}}}\left(x_{i}^{\prime} \equiv F^{l}\left(x^{\prime}\right)\right.$, is an interval type-1 set, as follows (11)-(13).

$F^{l}\left(x^{\prime}\right)=\left[\underline{f^{l}}\left(x^{\prime}\right), \bar{f}^{l}\left(x^{\prime}\right)\right] \equiv\left[\underline{f}^{l}, \bar{f}^{l}\right]$

where $\underline{f}^{l}\left(x^{\prime}\right)=\underline{\mu}_{\widetilde{F}_{1}}\left(x_{1}^{\prime}\right) * \cdots * \underline{\mu}_{\widetilde{F}_{p}{ }^{l}}\left(x_{p}^{\prime}\right)$

and $\bar{f}^{l}\left(x^{\prime}\right)=\bar{\mu}_{\widetilde{F}_{1}^{l}}\left(x_{1}^{\prime}\right) * \ldots * \bar{\mu}_{\widetilde{F}_{p}{ }^{l}}\left(x_{p}^{\prime}\right)$ 

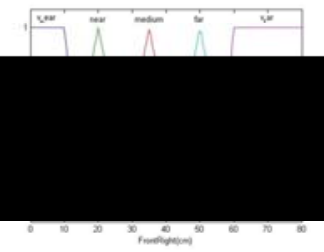

(a)
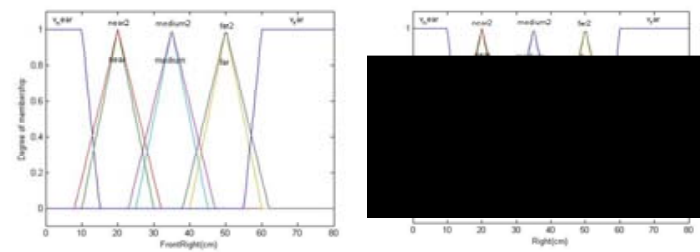

(b)
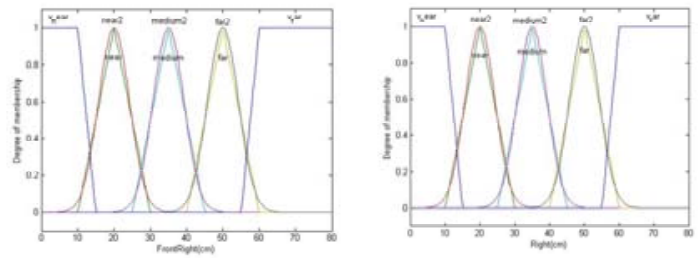

(c)

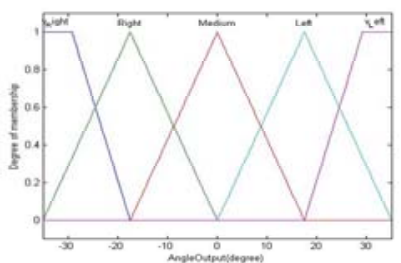

(d)

Fig. 7(a)traditional (b) type 2 (c) variation type 2 input membership function, (d). Traditional output membership function

4)Type Reducer: The type-1 fuzzy set is an interval set, we used center of sets type reduction, which is expressed as (14).

$$
\begin{aligned}
& Y_{\cos }(x)=\left[y_{l}, y_{r}\right] \\
& =\int y^{l} \in\left[y_{l}^{1}, y_{r}^{1}\right] \cdots \int y^{M} \in\left[y_{l}^{M}, y_{r}^{M}\right] \cdots \int f^{1} \in\left[f^{1}, \bar{f}^{1}\right] \cdots \\
& \int f^{M} \in\left[\underline{f}^{M}, \bar{f}^{M}\right] / \frac{\sum_{i=1}^{M} f^{i} y^{i}}{\sum_{i=1}^{M} f^{i}}
\end{aligned}
$$

The interval set corresponds to the centroid of the type-2 interval consequents $\widetilde{G}^{i}$

$C_{\widetilde{G}^{i}}=\int \theta_{1 \in J_{y 1}} \cdots \int \theta_{N \in J_{y N}} / \frac{\sum_{i=1}^{N} y_{i} \theta_{i}}{\sum_{i=1}^{N} \theta_{i}}=\left[y_{l}^{i} y_{r}^{i}\right]$

The values of $y_{l}$ and $y_{r}$ define the output interval of type- 2 fuzzy system, as follows equation (16) and (17).

$$
\begin{aligned}
& y_{l}=\frac{\sum_{i=1}^{M} f_{l}^{i} y_{l}{ }^{i}}{\sum_{i=1}^{M} f_{l}^{i}} \\
& y_{r}=\frac{\sum_{i=1}^{M} f_{r} y^{i} y_{r}}{\sum_{i=1}^{M} f_{r}{ }^{i}}
\end{aligned}
$$

5)Defuzzifier: The defuzzified output of an interval type-2 fuzzy logic system is as follows

$$
y(x)=\frac{y_{l}+y_{r}}{2}
$$

\section{B. Variation of Type-2 Fuzzy Logic System}

We propose a variation of type-2 fuzzy logic system in which the fuzzy membership has two different types: one is triangular type and the other is Gaussian type. Fig. 7 shows the variation of type-2 fuzzy membership function, traditional, and type-2 normal type. Fig. 7(a), (b), (c) are traditional, type2 , and variation type-2 fuzzy set of membership function of designed inputs, and Fig. 7(d) is designed outputs, respectively. In order to make a corresponding set, so we used traditional set for output. The sensing range is returned from front, front right, and right side as shown in Figure 8.

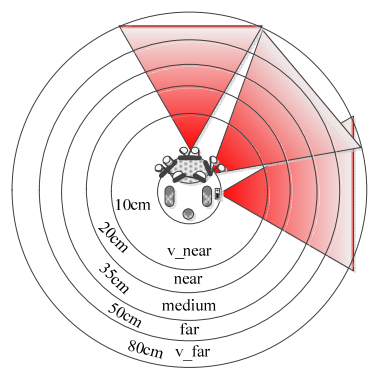

Fig. 8. The sensing range of the sensors

Therefore, in Table II we design 25 rules for fuzzy inference system through iterative tests.

\section{Processing Situations of Wall Following}

When the robot moves in a simply connected environment with corners, we assume that a wall can be perceived by one or more sensors, and at least one sensor can perceive one or more walls during robot motion. For example, when a mobile robot tries to pass around a corner, the robot motion is not too fast so that the corner is within field of view for all instants of time. With the assistance of sensors to correctly identify the situations the robot faces, we design a fuzzy controller of wall following in three main situations: (i) flat wall or smooth wall shown in Fig. 8, (ii)sharp corner shown in Fig. 9, and (iii)limit corner shown in Fig. 10. In these figures, the blue scan light is sonar and the red scan light is IR sensor, and there are three scan ranges as fuzzy inputs. The sensors should assist the robot to correctly identify the situation it faces. The first 
Table II

\begin{tabular}{|c|c|c|c|}
\hline & \multicolumn{2}{|c|}{ IF } & THEN \\
\hline Variable & FrontRight & Right & $\theta$ \\
\hline Rule1 & v_near & v_near & v_Left \\
\hline Rule2 & v_near & near & v_Left \\
\hline Rule3 & v_near & medium & v_Left \\
\hline Rule4 & v_near & far & v_Left \\
\hline Rule5 & v_near & v_far & Left \\
\hline Rule6 & near & v_near & V_Left \\
\hline Rule7 & near & near & v_Left \\
\hline Rule8 & near & medium & v_Left \\
\hline Rule9 & near & far & v_Left \\
\hline Rule10 & near & v_far & Left \\
\hline Rule11 & medium & v_near & Medium \\
\hline Rule12 & medium & near & Medium \\
\hline Rule13 & medium & medium & Medium \\
\hline Rule14 & medium & far & Medium \\
\hline Rule15 & medium & v_far & Medium \\
\hline Rule16 & far & v_near & Right \\
\hline Rule17 & far & near & Right \\
\hline Rule18 & far & medium & Medium \\
\hline Rule19 & far & far & Right \\
\hline Rule20 & far & v_far & Right \\
\hline Rule21 & v_far & v_near & Right \\
\hline Rule22 & v_far & near & Right \\
\hline Rule23 & v_far & medium & Medium \\
\hline Rule24 & v_far & far & Right \\
\hline Rule25 & v_far & v_far & Right \\
\hline
\end{tabular}

situation could appear in one of six situations for the design of rule base, which is shown in Fig. 9. Once the front sensing detects a safety distance, the mobile robot turns around to left. If both the front right and right sensing detect a medium just like the above situation of Fig. 9(a), the mobile robot moves along the wall. For the situation of Fig. 9( b) that the mobile robot is too close to the wall, we can see the rule 1 to rule 10 of the rule table Table 2 which turn left large or normal are applicable. Once the front sensing detects safety distance like situation Fig. 9(c), then the mobile robot turns to left. At the same time, the mobile robot must turn right to find a right wall when it meets the situations of Fig. 9(d), (e), and (f). The second part is when the mobile robot detects a sharp corner, which it is possible that the mobile robot loses sensing of the corner if moving too fast. This could appear in two situations, which is shown in Fig. 10. We determined how to turn around a sharp corner by sensing range. According to the situation of Fig. 10(a), the robot determined to turn right along with the sharp corner, and we can see the rule 16 to rule 18 of the rule table Table 2 are applicable. However, dealing with the situation of Fig. 10(b) is referred to rule 5 and rule 10 of the rule table Table 2 , and revised slightly to left. The third part could appear only in a situation, which is shown in Fig. 11. When this situation occurs, the robot must turn around to the left, and keep operating on the first and second situations.

In summary, the rule base of the fuzzy controller that copes with three possible situations of wall following is shown in Table III.

\section{Human Detection}

We equipped two human detection sensors on mobile robot for detecting the appearance of an invader on indoor environment. Fig. 12 demonstrates a scenario of the invader detection. The

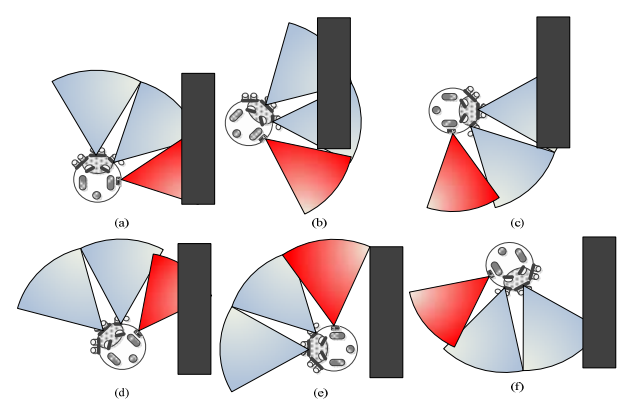

Fig. 9. First situation. The flat wall is encountered in six situations

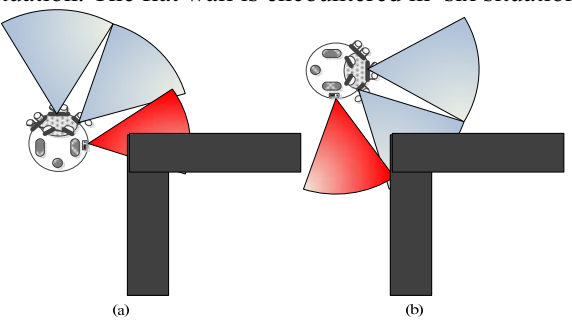

Fig. 10. Second situation. The sharp corner appears in two situations

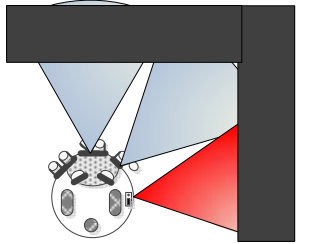

Fig. 11. Third situation. The situation of limit corner

Table III

\begin{tabular}{|c|c|}
\hline Situations & Rules \\
\hline The first part (on the flat wall) Fig. 9 \\
\hline (a) & $11 \sim 15$ \\
\hline (b) & $1 \sim 10$ \\
\hline (c) & Left Rotation \\
\hline (d) & $16 \sim 23$ \\
\hline (e) & 24,25 \\
\hline (f) & 24,25 \\
\hline The second part (at the sharp corner) Fig. 10 \\
\hline (a) & $21,22,24,25$ \\
\hline (b) & $13 \sim 15$ \\
\hline The third part (at the limited wall) Fig. 11 \\
\hline \multicolumn{2}{|c|}{ Left Rotation } \\
\hline
\end{tabular}

mobile robot autonomously navigates on an unknown indoor environment, and perform wall following of until it finds out an invader and starts detecting the human from human detection sensors. The robot will sound alarm, take a photo and send it to the remote user, then keep moving forward using wall following. Fig. 12 shows the flow chart of our surveillance system using right wall following. 


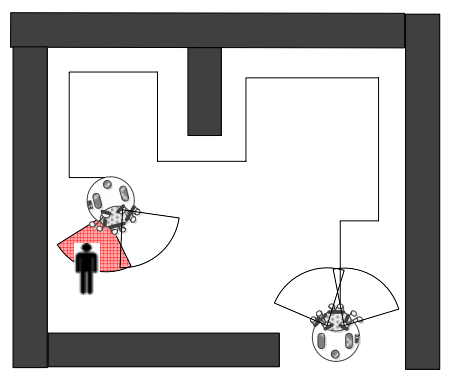

Fig. 12. A scenario of human detection using wall following The red scan light denotes detecting an invader and the mobile robot will sound alarm, take a photo and keep moving.

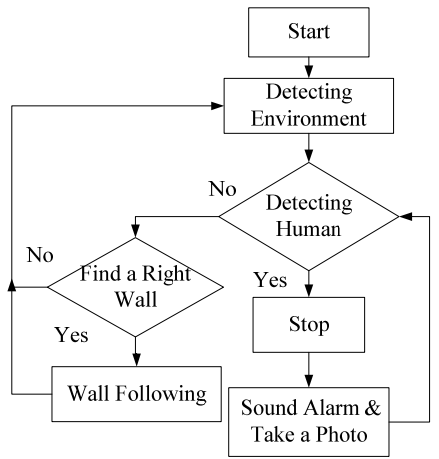

Fig. 13. The flow chart of surveillance system using right wall following

\section{EXPERIMENTAL RESULTS AND DISCUSSIONS}

\section{A. Simulation}

The variation of type-2 fuzzy controller will be applied to the mobile robot, and the simulation result shown in Fig. 13 thatthe performance is well. Fig. 14 shows the simulation results of three types of the fuzzy logic system, (a) is traditional, (b) is type-2 fuzzy, and we can see (c) is balanced which thus is used on mobile robot controller in the experiment.

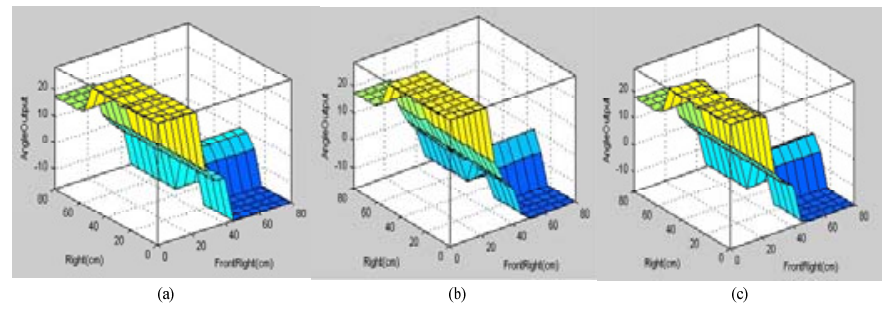

Fig. 14. The simulation with traditional (a), type-2 (b), and variation type-2 (c) fuzzy system

\section{B. Experiment on a complex polygon terrain}

We perform another experiment of surveillance on a complex polygon terrain, and the resulting trajectory of navigation shown in Fig. 15(a). The mobile robot performs wall following and detects an invader opening a door, and the mobile robot sound alarm that shown in Fig. 15(b). The mobile robot continued to perform patrol of wall following task when the invader leaves until stop command shown in
Fig. 15(c). This experiment is shown in Fig. 16 with the wheel velocities shown in Fig. 17. The strong shock of human detection sensor feedback voltage shown in the red line of Fig. 18 indicates the appearance of a person in the way. The motion control strategy proposed is powered with DC motor battery and DSP board, and the voltage fitting consumption is shown in Fig. 19. Since the mobile robot follows a smoother trajectory using our fuzzy differential velocity control approach, the energy assumption is more economical.

\section{Discussions}

In the experiment, human detection sensor provides reliable and effective detection of the appearance of human which interferes the robot path, enabling a successful wall following of surveillance mobile robot using interval type-2 fuzzy logic based navigation. In our experiment, only one human is detected at a time, and multiple humans could be detected if each appears separately at different time.
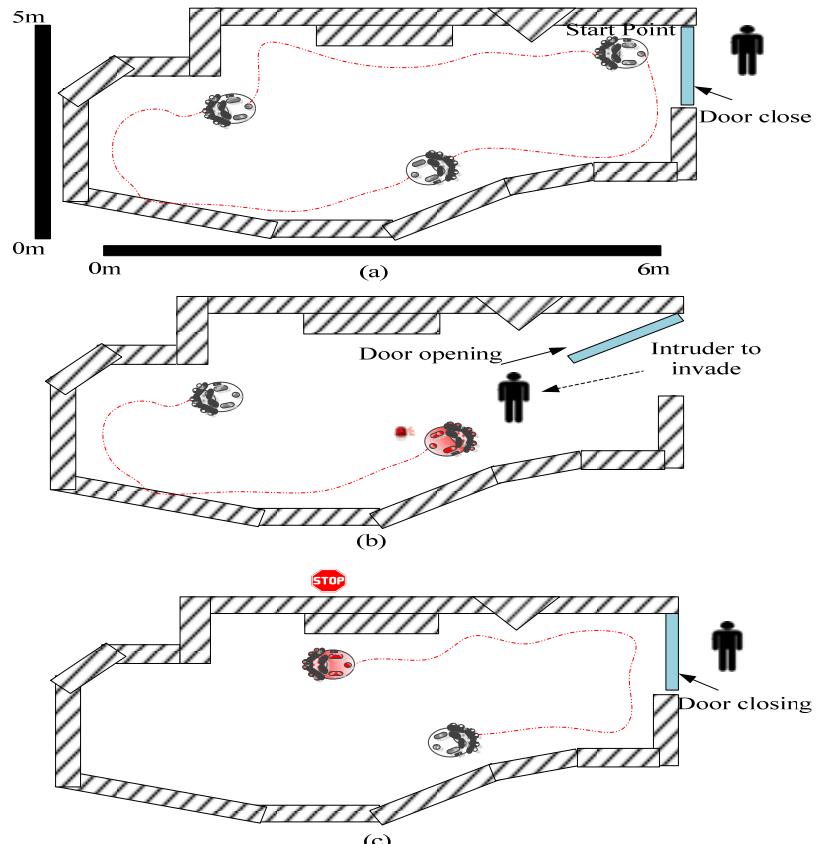

Fig. 15. Wall following with human detection on a complex polygon terrain (a)The trajectory of wall following (b)detects an invader.(c) The robot continued wall following on a complex polygon terrain

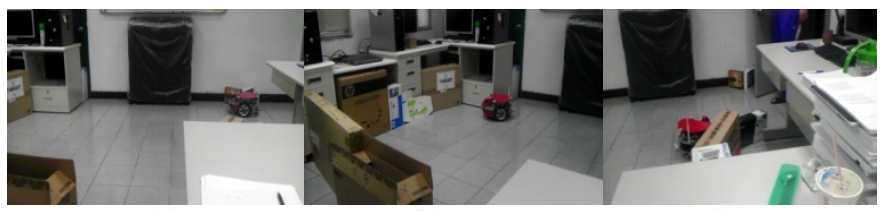

(a)

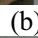

(c)

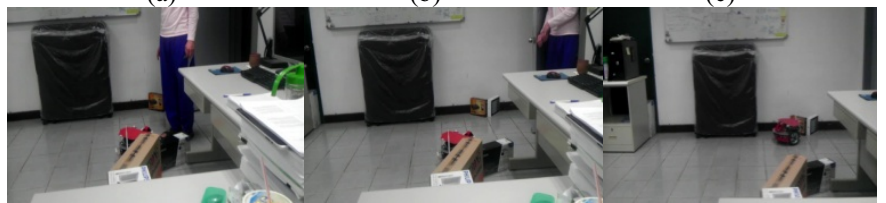

(d)

(e)

(f)

Fig. 16. Snapshots of experiment on wall following with human detection on 
a complex polygon terrain

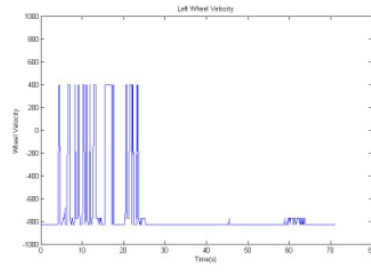

(a)Left wheel speed

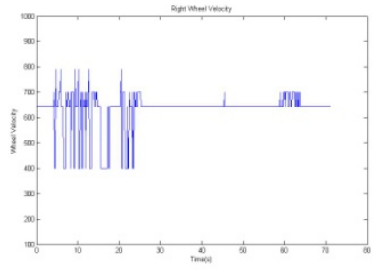

(b)Right wheel speed
Fig. 17. The wheel velocities

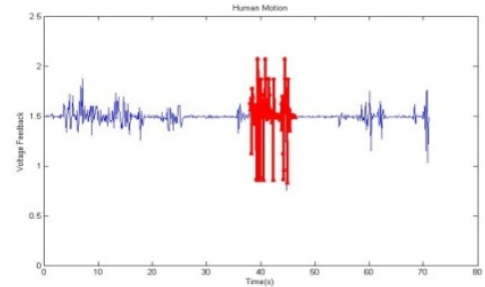

Fig. 18. The human detection sensor feedback voltage to mobile robot

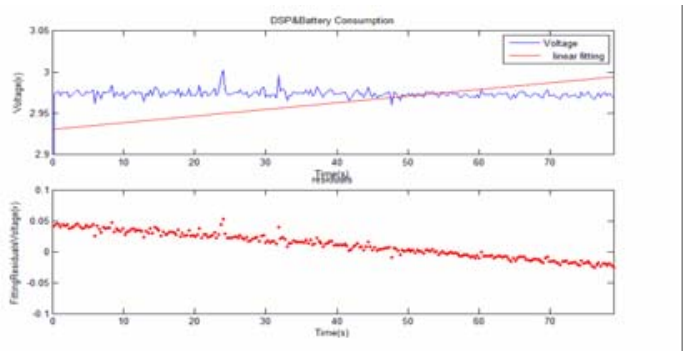

Fig. 19 The DSP board and DC motors battery consumption

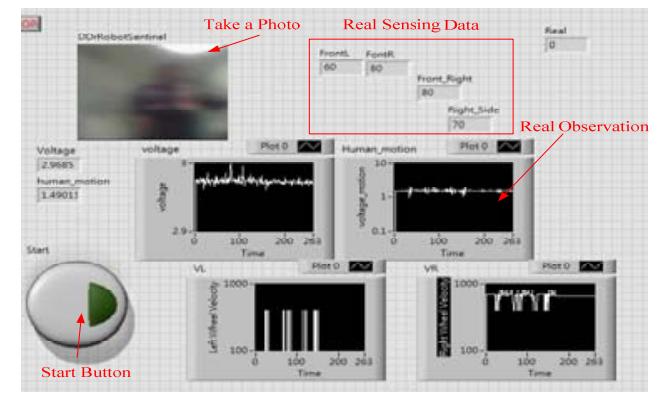

Fig. 20. The GUI of control panel using Labview

\section{CONCLUSION}

In this study, an intelligent differential velocity control scheme based on interval type-2 fuzzy logic theory is proposed to support a commercially available mobile robot platform equipped with range and human detection sensors for wall following with human detection. The control system uses the Labview software as graphical user interface (GUI) to design for on-line monitoring the mobile robot navigation, as shown in Fig. 20. This provides the remote user the real time observed data of local information from sensors of mobile robot navigation experiment as well as visualization of simulations results. We conduct two wall following tasks of mobile robot in real unknown environments, which also demonstrates the presented satisfactory result of human detection in the way of following the wall. The surveillance mobile robot is demonstrated effective to work well in our testing experiment involving the abrupt appearance of human. Future work is the study of human following and the design of human following controller.

\section{REFERENCES}

[1] P. Van Turennout, G. Honderd and L. J. Van Schelven, "Wallfollowing control of a mobile robot," IEEE International Conference on Robotics and Automation, pp. 280-285, 1992.

[2] W. Tsui, M. S. Masmoudi, F. Karray, I. Song and M. Masmoudi, "Softcomputing-based embedded design of an intelligent wall/lane-following vehicle," IEEE/ASME Transactions on Mechatronics, vol.1, no.1, pp.125-135, 2008

[3] C.-H. Hsu and C.-F. Juang, "Evolutionary Robot Wall-Following Control Using Type-2 Fuzzy Controller With Species-DE-Activated Continuous ACO," IEEE Transactions on Fuzzy Systems , vol.21, no. 1, pp.100-112, 2013

[4] A. G. Lamperski, O. Y. Loh, B. L. Kutscher and N. J. Cowan,'Dynamical wall following for a wheeled robot using a passive tactile sensor,"IEEE International Conference on Robotics and Automation, pp. 3838-3843, 2005.

[5] M.Hoy, "A Method of Boundary Following by a Wheeled Mobile Robot Based on Sampled Range Information." Journal of Intelligent \& Robotic Systems, vol. 72, no. 3-4, pp.463-482, 2013.

[6] Y. Ando and S. Yuta, "Following a wall by an Autonomous Mobile Robot with a Sonar-Ring," Proc. Conf. on Vehicle Navigation and Information System, pp. 206-213, Aug. 1995.

[7] A. De and D.E. Koditschek, "Toward dynamical sensor management for reactive wall-following," 2013 IEEE International Conference on Robotics and Automation , pp. 2400-2406, 2013.

[8] J. Borenstein and Y. Koren," Obstacle Avoidance with Ultrasonic Sensors." IEEE Transactions on Robotics and Automation, vol. 4, no.2, pp. 213-218, 1988.

[9] CW Lo, Kunlin Wu, YC Lin and JS Liu, "An intelligent control system for mobile robot navigation tasks in surveillance," 2nd International Conference on Robot Intelligence Technology and Applications, Denver, Colorado, USA, December 2013.

[10] T.H. Lee, H.K. Lam, F.H.F. Leung and P.K.S. Tam,” A Practical Fuzzy Logic Controller for The Path Tracking of Wheeled Mobile Robots." Application Note IEEE Control Systems Magazine, pp. 60-65, 2003.

[11] K.S. Chang and J.S. Choi," Automatic Vehicle Following Using the Fuzzy Logic." Proc. Conf. on Vehicle Navigation and Information System, pp. 206-213, Aug. 1995.

[12] R. Carelli and E. Oliveira Freire,"Corridor navigation and wallfollowing stable control for sonar-based mobile robots," Robotics and Autonomous Systems, vol.45, no.3, pp. 235-247, 2003.

[13] Benjamin Choi, Cetin Mericli, Joydeep Biswas, and Manuela Veloso, "Fast Human Detection for Indoor Mobile Robots Using Depth Images," IEEE International Conference on Robotics and Automation, August, 2013.

[14] http://www.drrobot.com/products/item_downloads/DHM5150_1.pdf

[15] Oscar Castillo, Patricia Melin, "A review on the design and optimization of interval type-2 fuzzy controllers." Applied Soft Computing 12 (2012) 1267 - $1278 \mathrm{G}$.

[16] O. Castillo and P. Melin, Type-2 Fuzzy Logic: Theory and Applications Springer-Verlag, 2008.

[17] Barili, A. Ceresa, M. Parisi, C, "Energy-saving motion control for an autonomous mobile robot," IEEE International Symposium on Industrial Electronics, pp. 674 - 676, 1995.

[18] Guido M. Sanchez, Leonardo L. Giovanini, “Autonomous navigation with deadlock detection and avoidance," Inteligencia Arti_cial 17(53) (2014), 13-23. 